\title{
Late gadolinium enhancement by cardiovascular magnetic resonance is complementary to left ventricle ejection fraction in predicting prognosis of patients with stable coronary artery disease
}

\author{
Oronzo Catalano ${ }^{1 *}$, Guido Moro², Mariarosa Perotti ${ }^{1}$, Mauro Frascaroli², Monica Ceresa', Serena Antonaci ${ }^{3}$, \\ Paola Baiardi ${ }^{4}$, Carlo Napolitano ${ }^{5,6}$, Maurizia Baldi ${ }^{2}$ and Silvia G Priori ${ }^{1,7}$
}

\begin{abstract}
Background: Late gadolinium enhancement (LGE) cardiovascular magnetic resonance (CMR) predicts adverse prognosis in patients with stable coronary artery disease (CAD). However, the interaction with conventional risk factors remains uncertain. Our aim was to assess whether the extent of LGE is an independent predictor of adverse cardiac outcome beyond conventional risk factors, including left ventricle ejection fraction (LVEF).

Methods: We enrolled 376 patients (88\% males, $64 \pm 11$ years) with stable CAD, who underwent LGE assessment and a detailed conventional evaluation (clinical and pharmacological history, risk factors, ECG, Echocardiography). During a follow-up of $38 \pm 21$ months, 56 events occurred (32 deaths, 24 hospitalizations for heart failure).

Results: LGE and LVEF showed the strongest univariate associations with end-points (HR: 13.61 [95\%C.I.: 7.3225.31] for LGE $\geq 45 \%$ of LV mass; and 12.34 [6.80-22.38] for LVEF $\leq 30 \%$; $p<0.0001$ ). Multivariate analysis identified baseline LVEF, loop diuretic therapy, moderate-severe mitral regurgitation and pulmonary hypertension as significant predictors among conventional risk factors. According to a step-wise approach, LGE showed strong association with prognosis as well (5.25 [2.64-10.43]; $p<0.0001)$. LGE significantly improved the model predictability (chi-square 239 vs 221, F-test $p<0.0001$ ) with an additive effect on the prognostic power of LVEF, which however retained its prognostic power (4.89 [2.50-09.56]; $p<0.0001$ ). Patients with LGE $\geq 45 \%$ and/or $\mathrm{LVEF} \leq 30 \%$ had much worse prognosis compared to patients without risk factors (annual event rates of $43 \%$ vs 3\%; $p<0.0001$ ). Interestingly LGE was a significant predictor when all cause mortality was analyzed as the only endpoint.
\end{abstract}

Conclusions: This study demonstrates that LGE assessed by CMR is a robust independent non-invasive marker of prognosis in stable CAD patients. LGE can integrate the available metrics to substantially improve risk stratification.

\section{Background}

Late gadolinium enhancement (LGE), assessed with cardiovascular magnetic resonance (CMR), has high sensitivity and specificity to detect and quantify fibrotic tissue due to myocardial infarction (MI) [1-3].

Previous studies suggested that LGE predicts adverse prognosis in patients with stable coronary artery disease (CAD) [4-9]. Since it also predicts unfavourable left

\footnotetext{
* Correspondence: oronzo.catalano@fsm.it

'Divisione di Cardiologia, IRCCS Fondazione Salvatore Maugeri, via Maugeri 6, Pavia, Italy

Full list of author information is available at the end of the article
}

ventricle (LV) remodelling after acute MI [10], LGE might be considered a metric of LV pump dysfunction alternative to ejection fraction (EF) or end-systolic volume (ESV). Pathophysiologic correlation between LGE and LVEF and equivalence of informative content seem to be supported by studies showing that LGE inclusion in multivariate models often leads to substantial blunting of the well known prognostic power of LVEF [5,6]. Interestingly, however, this evidence has not been confirmed by other studies in which both scar extent and LVEF seem to have an independent prognostic value $[9,11]$. Moreover, LGE retains a prognostic significance in the subset of patients 
with reduced LVEF [7]. Finally, conflicting results have been found in patients with stable CAD and unrecognized MI, with LGE prognostic power being incremental or alternative to LVEF at multivariate analysis $[4,8]$. Thus, the prognostic significance of LGE seems to be complex and not yet completely elucidated.

The aim of our study was to assess whether, in a large well-characterized population of patients with known or suspected stable CAD the extent of LGE is an independent predictor of adverse outcome in the long-term beyond conventional risk factors, in particular LVEF.

\section{Methods}

\section{Study population and design}

We performed a single centre observational prospective study. Inclusion criteria: consecutive patients clinically referred for CMR from January 2002 to December 2006, either with definite diagnosis or with a history suggesting stable CAD. Exclusion criteria: recent acute coronary syndrome (within 6 weeks), previous hospitalization for heart failure (NYHA class IV or need of infusive therapy) and signs of myocarditis, infiltrative or hypertrophic cardiomyopathy and pericardial disease. Patients underwent detailed clinical and instrumental risk stratification and were prospectively followed up. The study was approved by the Fondazione Maugeri ethical committee and informed consent was obtained from patients.

\section{Conventional risk assessment}

Before CMR execution patients' clinical history was collected, including anthropometric data, atherosclerotic risk factors profile, any documented CAD history, coronary angiography, NYHA class and pharmacological records. An ECG was also recorded the same day of CMR. ECGs were automatically analysed about heart rate, PR interval, QTc interval and QRS duration (E-Scribe System, Mortara Rangoni Europe), and interpreted by a single blinded reader (O.C.) with regard to rhythm, signs of LV hypertrophy, left and right bundle branch block, ST depression, negative $\mathrm{T}$ waves and $\mathrm{Q}$ waves presence. Patients underwent an echocardiographic examination within few days from CMR with up-to-date equipments (Sonos 5500, Hewlett Packard; Sequoia 512, Acuson; Vivid 7, General Electric). We evaluated dimensions, mass, segmental/global contractility and diastolic function of LV, mitral regurgitation, RV dimension and function, and pulmonary artery pressure (evaluation criteria for ECG and Echo are provided in additional file 1).

\section{CMR and LGE assessment}

CMR was performed with a 1.0-T scanner with a 20mTgradient (Magneton Harmony, Siemens, Erlangen, Germany) and a phased-array cardiac coil. LGE was assessed by inversion-recovery turboFLASH sequences
(TE $2.6 \mathrm{msec}$, FA $8^{\circ}$, inversion time $260-360 \mathrm{msec}$, matrix $96 \times 256$; FOV $400 \mathrm{~mm}$ ), $7-8 \mathrm{~min}$ after $0.15-0.20 \mathrm{mmol} / \mathrm{kg}$ intravenous injection of gadolinium (Magnevist; Schering, Berlin, Germany; Multihance, Bracco, Milan, Italy). Multiple $8 \mathrm{~mm}$ thick short-axis slices (usually 8-10) with appropriate interslice space (usually $2 \mathrm{~mm}$ ) were used for a full coverage of LV. Transmural extent of LGE was scored by consensus of two experienced readers (O.C., G.M.), using a five point scale: $0=$ no LGE, $1=1-25 \%, 2=26-50 \%, 3=51-75 \%$ and $4=76-100 \%$ of wall thickness [12]. Standard 17-segments segmentation of LV was used and total LGE burden in percent of total LV mass was calculated ([total score*100]/[17*4]). Maximal transmural extent and spatial extent, that is maximal LGE score and the number of affected segments, were considered too.

\section{Follow-up}

Follow up visits were performed at our Centre every 1-24 months, depending on the clinical severity. Transtelephonic follow-up was collected only for those patients whose last visit date was antecedent 6 month database closure (March 2009).

Primary outcome measure was a composite clinical end-point of all-cause mortality and new onset heart failure (HF) requiring hospitalization (NYHA class IV or need of infusive therapy). If the patients were admitted to a hospital other than our institution we retrieved the hospital record to confirm diagnosis, clinical parameters and outcome.

Myocardial revascularization procedures occurring after CMR were registered as well, to evaluate any modulating effect of myocardial revascularization on prognostic value of LGE.

\section{Statistics}

Categorical variables were expressed as counts and percentage, continuous variables as mean \pm standard deviation. Two sided $P<0.05$ was the significance level for hypothesis testing and SPSS Statistics 18.0 was the statistical package we used.

Differences at baseline between patients with and without events were tested with Pearson Chi-Square or Fisher's exact test in case of categorical variables and Student's $t$-test or Mann-Whitney $U$-test in case of continuous variables.

Univariate hazard ratios were calculated by Cox analysis after converting continuous variables into dichotomous variables; cut-offs were taken from the literature. Specifically dichotomization of LVEF was made according to the more conservative SCDHeft cut-off (30\%). If established cut-offs were lacking, we used the 75th and the 95th percentiles of the entire study population. Proportional 
Table 1 Baseline characteristics and differences between patients with and without events in the follow-up

\begin{tabular}{|c|c|c|c|c|}
\hline & All patients & Event free $(n=320)$ & With events $(n=56)$ & P Value* \\
\hline \multicolumn{5}{|l|}{ ANTHROPOMETRY } \\
\hline Age (y) & $64 \pm 11$ & $63 \pm 11$ & $68 \pm 10$ & 0.003 \\
\hline Sex $(m)$ & $292(78 \%)$ & $248(76 \%)$ & $44(79 \%)$ & 0.859 \\
\hline Body mass index & $26 \pm 4$ & $26 \pm 4$ & $26 \pm 4$ & 0.300 \\
\hline \multicolumn{5}{|l|}{ CAD RISK FACTORS } \\
\hline Familiar history of CAD & $170(45 \%)$ & $143(45 \%)$ & 27 (48\%) & 0.625 \\
\hline Smoking habit & $220(59 \%)$ & $179(56 \%)$ & $41(73 \%)$ & 0.015 \\
\hline Diabetes & 77 (21\%) & $66(21 \%)$ & $11(20 \%)$ & 0.867 \\
\hline Hypertension & $218(58 \%)$ & $185(58 \%)$ & $33(59 \%)$ & 0.876 \\
\hline Hypercholesterolemia & $214(57 \%)$ & $181(57 \%)$ & $33(59 \%)$ & 0.742 \\
\hline \# risk factors & $2.4 \pm 1.1$ & $2.4 \pm 1.1$ & $2.6 \pm 1.1$ & 0.159 \\
\hline \multicolumn{5}{|l|}{ CLINIC HISTORY } \\
\hline Previous CAD diagnosis & $332(88 \%)$ & $277(87 \%)$ & $55(98 \%)$ & 0.012 \\
\hline Previous myocardial infarction & $246(65 \%)$ & $202(63 \%)$ & $44(79 \%)$ & 0.025 \\
\hline NYHA classification (III class) & $22(6 \%)$ & $11(3 \%)$ & $11(20 \%)$ & $<0.0001$ \\
\hline Revascularization in the follow-up & 79 (21\%) & $73(23 \%)$ & $6(11 \%)$ & 0.040 \\
\hline \multicolumn{5}{|l|}{ PHARMACOLOGICAL THERAPY } \\
\hline$\beta$-blockers & $289(77 \%)$ & $448(78 \%)$ & $41(73 \%)$ & 0.483 \\
\hline $\mathrm{Ca}^{++-}$antagonist & $76(20 \%)$ & $62(19 \%)$ & $14(25 \%)$ & 0.334 \\
\hline Nitrates & $159(42 \%)$ & $136(43 \%)$ & $23(41 \%)$ & 0.842 \\
\hline Loop diuretics & $135(36 \%)$ & $95(30 \%)$ & $40(71 \%)$ & $<0.0001$ \\
\hline Aldosterone antagonist & $51(14 \%)$ & $30(9 \%)$ & $21(38 \%)$ & $<0.0001$ \\
\hline ACE-inhibitors/AT ${ }_{1}$-receptors antagonist & $304(81 \%)$ & $257(80 \%)$ & 47 (84\%) & 0.526 \\
\hline ASA & $319(85 \%)$ & $275(86 \%)$ & $44(79 \%)$ & 0.156 \\
\hline Statins & $280(75 \%)$ & $240(75 \%)$ & $40(71 \%)$ & 0.572 \\
\hline Anticoagulant & $33(9 \%)$ & $18(6 \%)$ & $15(27 \%)$ & $<0.0001$ \\
\hline \multicolumn{5}{|l|}{ ECG } \\
\hline Heart rate (bpm) & $65 \pm 13$ & $64 \pm 12$ & $73 \pm 14$ & $<0.0001$ \\
\hline Non sinusal rhythm & $12(3 \%)$ & $7(2 \%)$ & $5(9 \%)$ & 0.021 \\
\hline QRS duration (msec) & $105 \pm 21$ & $103 \pm 19$ & $112 \pm 27$ & 0.022 \\
\hline QTc interval (msec) & $425 \pm 34$ & $421 \pm 32$ & $447 \pm 37$ & $<0.0001$ \\
\hline LV hypertrophy & $58(15 \%)$ & $50(14 \%)$ & $8(16 \%)$ & 0.942 \\
\hline LBB block & $59(16 \%)$ & $44(14 \%)$ & $15(27 \%)$ & 0.013 \\
\hline RBB block & $16(12 \%)$ & $12(12 \%)$ & $4(14 \%)$ & 0.612 \\
\hline ST segment depression & $46(8 \%)$ & $38(7 \%)$ & $8(13 \%)$ & 0.176 \\
\hline Negative T waves & 184 (49\%) & $151(47 \%)$ & $33(59 \%)$ & 0.105 \\
\hline Q waves & $164(44 \%)$ & $135(42 \%)$ & $29(52 \%)$ & 0.181 \\
\hline \multicolumn{5}{|l|}{ ECHOCARDIOGRAPHY } \\
\hline LV EDV $\left(\mathrm{ml} / \mathrm{m}^{2}\right)$ & $59 \pm 22$ & $57 \pm 20$ & $74 \pm 30$ & $<0.0001$ \\
\hline LV ESV $\left(\mathrm{ml} / \mathrm{m}^{2}\right)$ & $31 \pm 20$ & $28 \pm 16$ & $49 \pm 28$ & $<0.0001$ \\
\hline LV EF (\%) & $51 \pm 13$ & $53 \pm 12$ & $39 \pm 15$ & $<0.0001$ \\
\hline LV WMSI & $1.4 \pm 0.5$ & $1.4 \pm 0.4$ & $1.9 \pm 0.5$ & $<0.0001$ \\
\hline LV mass $(g)$ & $188 \pm 59$ & $186 \pm 57$ & $202 \pm 70$ & $<0.0001$ \\
\hline LV diastolic function ( $\geq$ pseudo-normal) & $44(12 \%)$ & $25(8 \%)$ & 19 (34\%) & $<0.0001$ \\
\hline
\end{tabular}


Table 1 Baseline characteristics and differences between patients with and without events in the follow-up (Continued)

\begin{tabular}{|c|c|c|c|c|}
\hline Mitral regurgitation ( $\geq$ moderate) & $56(15 \%)$ & $36(11 \%)$ & $20(36 \%)$ & $<0.0001$ \\
\hline Pulmonary hypertension & $34(9 \%)$ & $19(6 \%)$ & $15(27 \%)$ & $<0.0001$ \\
\hline RVIT dilatation & $17(5 \%)$ & $12(4 \%)$ & $5(9 \%)$ & 0.085 \\
\hline RV dysfunction & $38(10 \%)$ & $28(9 \%)$ & $10(18 \%)$ & 0.037 \\
\hline \multicolumn{5}{|l|}{ LATE GADOLINIUM ENHANCEMENT } \\
\hline Total burden (\% of LV mass) & $13 \pm 15$ & $10 \pm 12$ & $28 \pm 22$ & $<0.0001$ \\
\hline Spatial extent (\% of LV surface) & $22 \pm 22$ & $18 \pm 19$ & $42 \pm 29$ & $<0.0001$ \\
\hline Max transmural extent (\% of wall thickness) & $55 \pm 39$ & $51 \pm 39$ & $73 \pm 39$ & $<0.0001$ \\
\hline
\end{tabular}

$\mathrm{LV}=$ left ventricle; $\mathrm{LBB}=$ left bundle branch; $\mathrm{RBB}=$ right bundle branch; $\mathrm{MR}=$ mitral regurgitation; $\mathrm{RVIT}=$ right ventricle inflow tract.

* Pearson Chi-Square or Fisher's exact test (where appropriate) for categorical data; Student's t-test or Mann-Whitney test (\# risk factors, LV WMSI, late enhancement data) for numeric data.

hazard assumption was graphically tested using plots of the log estimated cumulative baseline hazard against time.

Conventional variables correlated with prognosis $(p<0.1)$ at a first multivariate analysis (step-wise forward selection, forceful introduction of LVEF), were used to build the final model in which LGE was introduced at the last step to test the hypothesis of its independent prognostic value on top of a conventional risk stratification approach. F-test for extra sum of square principle was applied to assess goodness of fit of the final model with respect to the conventional nested model. Annual event rate and death rate for patients at risk were calculated.

\section{Results}

Four-hundred-ten patients were referred to our unit for CMR assessment during the period of interest. Twentyseven $(7 \%)$ were excluded according to the exclusion criteria and seven (2\%) patients were lost at follow-up. Thus, 376 patients entered the study, with a definite diagnosis of CAD at the enrolment in 332 cases (88\%) and suspicion history in 44 (12\%). Patients were followed-up for $38 \pm 21$ months, during which there were 56 events (32 deaths, 24 new onset HF cases).

Main baseline characteristics are reported in Table 1. Overall the study cohort was characterized by high prevalence of male sex (88\%). History of previous MI was detected in two thirds of cases. Pharmacological treatment was characterized by high rates of betablockers, ACE-inhibitors, ASA and statins administration (70-85\%) with no significant differences among patients with and without events.

\section{Predictors of events}

Survival Cox univariate analyses showed that outcome was associated with several variables. As known from previous studies, the most powerful predictors of events were age, previous history of CAD, 3-vessel disease at coronary angiography, NYHA class, need for diuretic and anticoagulant therapy, heart rate, non sinus rhythm,
QRS complex duration, QTc interval, LV volumes, LVEF, LV wall motion score index, LV diastolic function, mitral regurgitation, pulmonary hypertension and right ventricle function. A revascularization procedure after the study enrolment was a protecting factor against the outcome. All LGE indexes were also strongly associated with prognosis with total burden, that is the amount of LGE in percent of total LV mass, showing the most powerful correlation. The 95th percentiles was the best cut-off and that was considered for further analysis. Univariate hazard ratios with $95 \%$ confidence intervals of all considered variables are shown in Table 2.

The step-wise inclusion of variable reaching the predefined univariate $\mathrm{p}$ value threshold $(p<0.1)$ into a multivariate Cox model in which LVEF was forcefully included, significantly improved the model predictability (chi-square 163 vs 157, F test: $p=0.033$ ) with respect to considering LVEF alone. However, only loop diuretic therapy (HR: 3.20 [95\% C.I.: $1.71-5.97$ ]; $p=0.003$ ), pulmonary hypertension (2.44 [1.27 -4.70$] ; p=0.008)$ and moderate-severe mitral regurgitation $(2.02$ [95\% CI: $1.06-3.85$ ]; $p=0.028)$ were independently associated with an adverse prognosis after considering LVEF (5.54 [2.85 - 10.78]; $p<0.0001)$.

\section{Prognostic role of late gadolinium enhancement}

Significant conventional variables as from the multivariate analysis were used to build the final model, that included LGE and showed LGE to be significantly associated with an adverse prognosis in terms of death or new onset HF. LGE introduction significantly improved the model fit (chi-square 238 vs 223 , F-test $p=0.0001$ ) with respect to conventional variables alone. Moreover, LGE was the strongest prognostic indicator with a 5.3 fold increase of event risk at follow-up. However, LVEF retained a comparable prognostic power with a 4.9 fold increase of event risk (HRs shown in Table 3). Similar results were obtained analysing only patients with MI history at the enrolment $(n=246)$, with a definite diagnosis of CAD at the 
Table 2 Unadjusted hazard ratios for death or new heart failure

\begin{tabular}{|c|c|c|c|}
\hline & $\begin{array}{l}\text { Unadjusted } \\
\text { HR }\end{array}$ & $\begin{array}{l}\text { 95\% Confidence } \\
\text { Interval }\end{array}$ & $P$ value \\
\hline \multicolumn{4}{|l|}{ ANTHROPOMETRIC } \\
\hline Age (75 y) & 1.70 & $0.92-3.17$ & 0.093 \\
\hline Male sex & 0.92 & $0.67-1.27$ & 0.614 \\
\hline Body mass index $>30$ & 1.04 & $0.49-2.20$ & 0.916 \\
\hline \multicolumn{4}{|l|}{ RISK FACTORS } \\
\hline Familiar history of CAD & 1.16 & $0.68-1.96$ & 0.588 \\
\hline Smoking habit & 2.22 & $1.23-4.00$ & 0.008 \\
\hline Diabetes & 0.88 & $0.45-1.70$ & 0.698 \\
\hline Hypertension & 1.00 & $0.59-1.70$ & 0.999 \\
\hline Hypercholesterolemia & 1.13 & $0.66-1.92$ & 0.664 \\
\hline \# risk factors $\geq 3$ & 1.58 & $0.93-2.69$ & 0.093 \\
\hline \multicolumn{4}{|l|}{ CLINIC } \\
\hline Previous CAD diagnosis & 8.76 & $1.21-63.3$ & 0.032 \\
\hline $\begin{array}{l}\text { Previous myocardial } \\
\text { infarction }\end{array}$ & 2.51 & $1.32-4.76$ & 0.005 \\
\hline NYHA classification $\geq 3$ & 5.81 & $2.99-11.3$ & $<0.0001$ \\
\hline $\begin{array}{l}\text { Revascularization in the } \\
\text { follow-up }\end{array}$ & 0.36 & $0.15-0.85$ & 0.019 \\
\hline \multicolumn{4}{|l|}{ THERAPY } \\
\hline$\beta$-blockers & 1.00 & $0.55-1.81$ & 0.997 \\
\hline $\mathrm{Ca}^{++-}$antagonist & 1.15 & $0.63-2.12$ & 0.644 \\
\hline Nitrates & 0.92 & $0.54-1.56$ & 0.753 \\
\hline Loop diuretics & 5.26 & $2.94-9.40$ & $<0.0001$ \\
\hline Aldosterone antagonist & 5.69 & $3.29-9.86$ & $<0.0001$ \\
\hline $\begin{array}{l}\text { ACE-inhibitors/AT } T_{1} \text {-receptors } \\
\text { antagonist }\end{array}$ & 1.45 & $0.71-2.96$ & 0.310 \\
\hline ASA & 0.69 & $0.36-1.30$ & 0.251 \\
\hline Statins & 0.94 & $0.53-1.68$ & 0.837 \\
\hline Anticoagulant & 6.38 & $3.49-11.7$ & $<0.0001$ \\
\hline \multicolumn{4}{|l|}{ ECG } \\
\hline Heart rate ( $\geq 75$ bpm) & 2.80 & $1.62-4.84$ & $<0.001$ \\
\hline Non sinusal rhythm & 4.19 & $1.67-10.54$ & 0.002 \\
\hline QRS duration & 2.62 & $1.47-4.70$ & 0.001 \\
\hline QTc interval & 4.34 & $2.53-7.46$ & $<0.0001$ \\
\hline LV hypertrophy & 1.00 & $0.47-2.11$ & 0.995 \\
\hline LBB block & 1.63 & $1.07-2.48$ & 0.024 \\
\hline RBB block & 1.30 & $0.62-2.76$ & 0.489 \\
\hline ST segment depression & 1.60 & $0.73-3.54$ & 0.245 \\
\hline Negative T waves & 1.76 & $1.03-3.00$ & 0.039 \\
\hline Q waves & 1.55 & $0.92-2.62$ & 0.102 \\
\hline \multicolumn{4}{|l|}{ ECHOCARDIOGRAPHY } \\
\hline LV EDV $\left(\geq 105 \mathrm{ml} / \mathrm{m}^{2}\right)$ & 4.66 & $2.34-9.29$ & $<0.0001$ \\
\hline LV ESV $\left(\geq 75 \mathrm{ml} / \mathrm{m}^{2}\right)$ & 8.95 & $4.55-17.60$ & $<0.0001$ \\
\hline $\operatorname{LV}$ EF $(\leq 30 \%)$ & 12.34 & $6.80-22.38$ & $<0.0001$ \\
\hline
\end{tabular}

Table 2 Unadjusted hazard ratios for death or new heart failure (Continued)

\begin{tabular}{|c|c|c|c|}
\hline LV WMSI ( $\geq 2.32)$ & 10.94 & $5.53-21.62$ & $<0.0001$ \\
\hline LV mass ( $\geq 310 \mathrm{~g})$ & 4.89 & $2.05-11.70$ & $<0.001$ \\
\hline $\begin{array}{l}\text { LV diastolic function ( } \geq \\
\text { pseudo-normal) } \dagger\end{array}$ & 7.03 & $4.00-12.38$ & $<0.0001$ \\
\hline $\begin{array}{l}\text { Mitral regurgitation }(\geq \\
\text { moderate) } t\end{array}$ & 4.67 & $2.68-8.13$ & $<0.0001$ \\
\hline Pulmonary hypertension & 4.86 & $2.68-8.80$ & $<0.0001$ \\
\hline RVIT dilatation & 1.45 & $0.92-2.30$ & 0.112 \\
\hline RV dysfunction & 5.17 & $2.48-10.80$ & $<0.0001$ \\
\hline \multicolumn{4}{|l|}{ CMR } \\
\hline LGE total burden $\geq 45 \% \S$ & 13.61 & $7.32-25.31$ & $<0.0001$ \\
\hline LGE total burden $\geq 20 \%$ \# & 6.62 & $3.86-11.38$ & $<0.0001$ \\
\hline LGE spatial Extent $\geq 68 \% \S$ & 9.27 & $4.86-17.66$ & $<0.0001$ \\
\hline LGE spatial Extent $\geq 16 \%$ \# & 4.96 & $2.60-9.45$ & $<0.0001$ \\
\hline LGE transmurality & 4.82 & $2.81-8.31$ & $<0.0001$ \\
\hline
\end{tabular}

$\mathrm{LV}=$ left ventricle; $\mathrm{LVEF}=$ left ventricle ejection fraction; $\mathrm{LBB}=$ left bundle branch; $\mathrm{RBB}=$ right bundle branch; $\mathrm{MR}=$ mitral regurgitation; $\mathrm{RVIT}=$ right ventricle inflow tract; LGE = late gadolinium enhancement.

$\dagger$ based on trans-mitral diastolic flow and pulmonary vein flow evaluation.

$\downarrow$ based on effective regurgitate orifice area.

$\S$ cut-off equal to the $95 \%$ percentile of the entire population.

\# cut-off equal to the $75 \%$ percentile of the entire population.

end of the study $(n=344)$ or using CMR derived LVEF; HRs of these analyses are provided in additional file 2.

Accordingly, LVEF less than 30\% and LGE total burden more than $45 \%$ of total LV mass (95th percentile of the study population) sharply stratified the population at risk. The absence of both risk factors identified patients at low risk $(n=340)$, with event-free survival of $95 \%$ at 1 year, $92 \%$ at 3 years and $86 \%$ at 5 years, and mean annual event rate of $3 \%$; conversely the presence of at least 1 of these risk factors identified patients at very high risk ( $\mathrm{n}=36)$ with event-free survival of $60 \%$ at 1 year, $31 \%$ at 3 years and $21 \%$ at 5 years, and a mean annual rate of $43 \%$ (Log-Rank test: $p<0.0001$ ). Ten out of 36 (28\%) patients at high risk would not have been identified without considering LGE, since their LVEF was greater than $30 \%$.

Finally we repeated the analysis using total mortality as the only endpoint and we found similar results. In this group LGE was associated with significantly increased risk of mortality (HR 3.78 [95\% CI: 1.46 - 9.75]; $p=0.006$ ). All-cause mortality showed survival of $97 \%$ at 1 year, $95 \%$ at 3 years and $92 \% 5$ years, and mean annual death rate of $2 \%$ in the group without risk factors; compared to survival of $82 \%$ at 1 year, $63 \%$ at 3 years and $53 \%$ at 5 years, and mean annual death rate of $14 \%$ in the group with risk factors $(p<0.0001)$. These findings are graphically shown by Kaplan-Meyer curves in the Figure 1. 
Table 3 Adjusted hazard ratios for death or new heart failure of the final model

\begin{tabular}{lccc}
\hline & $\begin{array}{c}\text { Adjusted } \\
\text { HR }\end{array}$ & $\begin{array}{c}\text { 95\% Confidence } \\
\text { Interval }\end{array}$ & P value \\
\cline { 2 - 4 } & 5.25 & $2.64-10.43$ & $<0.0001$ \\
LGE total burden & & & \\
LVEF ( $\leq 30 \%)$ & 4.89 & $2.50-9.56$ & $<0.0001$ \\
Pulmonary hypertension & 2.89 & $1.56-5.36$ & $<0.001$ \\
(sPAP $\geq 35$ mmHg) & & $1.54-5.55$ & 0.001 \\
\hline Loop diuretics therapy & 2.92 & & \\
\hline
\end{tabular}

$\mathrm{LGE}=$ late gadolinium enhancement; LVEF = left ventricle ejection fraction; $\mathrm{SPAP}=$ systolic pulmonary artery pressure.

\section{Discussion}

In the last decade LGE CMR has paved the way to a new era in the assessment of CAD [1]. Indeed, for the first time this technique allowed to pursue a direct detection and quantification of areas of irreversibly injured myocardium. Thus LGE enabled in vivo morphopathologic assessment complementary to that of myocardium functionally impaired but still viable (hibernating myocardium), postulated from previous studies [13]. Accordingly, LGE had the potential to become the technique of choice to study myocardial viability. Recent studies have confirmed this hypothesis by showing that (a) the lesser transmural extent of scar is, the more likely functional recovery of a segment will be after revascularization and (b) the amount of viable plus normal segments is the best predictor of global functional recovery $[12,14]$.

In survival studies the extent of LGE has emerged as the strongest prognostic factor in stable CAD patients with previous MI [5,6]. This correlation further refines the
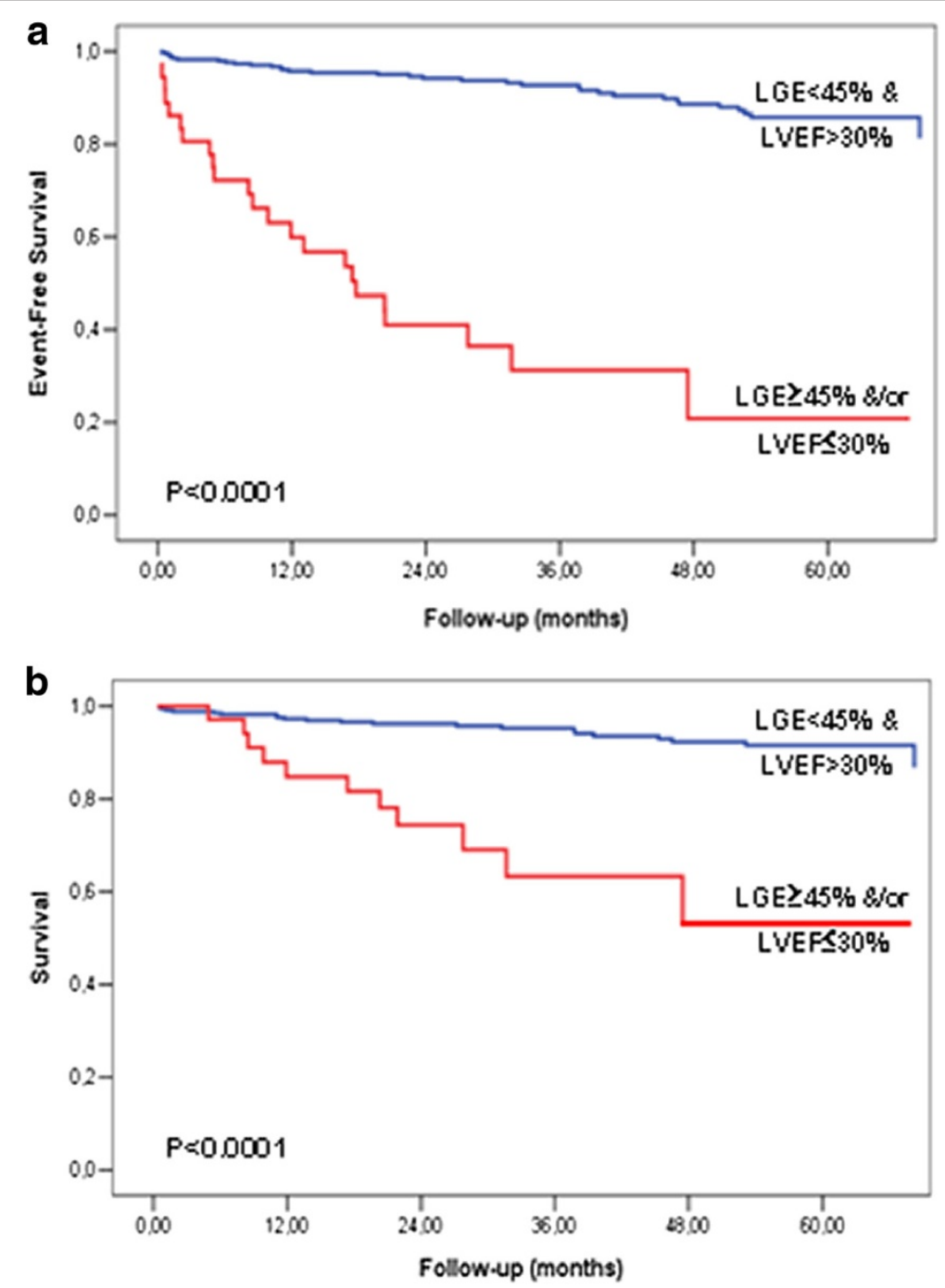

Figure 1 Prognostic value of late gadolinium enhancement and left ventricle ejection fraction. Kaplan-Meier survival analysis showing the cumulative incidence of death plus heart failure (panel a) or death alone (panel b). 
relationship between LVEF and prognosis because LGE directly reflects the amount of irreversibly injured myocardium. Accordingly, these studies seem to support the hypothesis that LVEF is no more significant once LGE is included in multivariate models. On the other hand, the idea of the predictive value of LVEF to be enclosed and overtaken by LGE assessment, is in conflict with a previous scintigraphy study in sudden death survivors and in a recent CMR study in hypertensive patients [9,11]. In these two studies, indeed, LVEF remained an independent predictor of events after the inclusion of scar dimension in a multivariate analysis. Thus the interaction between LGE and LVEF in stable CAD populations seems to be modulated by the selection of specific subsets of patients.

\section{Conventional risk stratification}

Our study was aimed at verifying these findings in a large group of unselected patients with stable CAD. For this reason we intentionally avoid to enrol a highly homogeneous study cohort by defining relatively loose entry criteria. In this setting, we attempted to test the hypothesis whether LGE is an independent predictor of adverse cardiac outcome in the context of a complex cohort undergoing a complete standard evaluation. We believe this study is representative of clinical referral of many outpatient CAD cardiology clinics.

We studied a group of 376 consecutive patients with stable CAD and optimized medical therapy, who were followed-up for an average time of 3 years. The conventional prognostic factor included in the study confirmed their association with adverse prognosis, thus further strengthening the evidence that our study group is representative of a general population with stable CAD. To remove redundant information a multivariate analysis was performed, which confirmed the expected strong prognostic power of LVEF and showed a significant contribution by only few other conventional variables. The latter did not include myocardial revascularization, even if it seemed to have a protective effect as from univariate analysis.

\section{Added value of late gadolinium enhancement}

On top of relevant conventional variables, we found that LGE was significantly and strongly associated with prognosis in a multivariate model (5.3 fold risk increase for LGE $>45 \%$ ). Even more importantly, the present study showed that LGE extent is complementary to LVEF in stratifying the risk of patients with stable CAD. A large scar, replacing more than $45 \%$ of total LV mass, well integrates with the generally accepted criterion of LVEF lower than $30 \%$ in identifying patients at high risk of death or HF. Accordingly, patients with either LGE $\geq 45 \%$ or $\operatorname{LVEF} \leq 30 \%$, have completely different prognosis if compared with patients without any of these risk factors, showing annual event rates of $43 \%$ versus 3\% and mortality rates of $14 \%$ versus $2 \%$, respectively. Notably, about $30 \%$ of patients at high risk would not have been identified without including LGE into the stratification process.

The result of the present study seems to reinforce the idea of LGE as a predictor of prognosis that adds to LVEF. In comparison to the studies of Roes et al. and Kelle et al., that came to opposite results, our study considered similar end-points but did not have scar presence as an inclusion criterion (approximately $70 \%$ of our patients were LGE positive). Accordingly, on average scar dimension was smaller (13\% vs $19-20 \%$ of total mass) and EF higher (51 vs $43-44 \%$ ). Thus, it may be hypothesized that the pre-selection of patients with a scar among those with stable CAD may influence the interaction between LGE and LVEF in the prediction of future events.

Understanding the relative relevance of LGE and LVEF is not a purely theoretical issue. Indeed, in patients with stable CAD reduced LVEF is a pre-requisite for important therapeutic decisions, such as cardiac resynchronization therapy (CRT) or automatic implantable cardioverterdefibrillator (AICD). Thus, will LGE be confirmed a stronger predictor than LVEF, the management of patients with stable CAD could be substantially changed

\section{Conclusions}

Our study confirms and refines the evidence of LGE as strong prognostic factor in unselected patients with stable CAD, showing a complementary role with respect to LVEF. Although further studies are warranted to assess the usefulness of LGE as selection criterion for major therapeutic decision such as CRT or AICD, findings of the present study promote the inclusion of LGE into current clinical management of patients with stable CAD, especially of those with reduced LVEF at echocardiography.

\section{Additional files}

Additional file 1: Table 1. Evaluation criteria of ECG and

Echocardiography. $\mathrm{LV}=$ left ventricle; $\mathrm{LBB}=$ left bundle branch; $\mathrm{RBB}=$ right bundle branch; $\mathrm{MR}=$ mitral regurgitation; $\mathrm{SPAH}=$ systolic pulmonary artery hypertension; RV = right ventricle; RVIT = right ventricle inflow tract; TAPSE = tricuspid anular plane systolic excursion * based on trans-mitral diastolic flow and pulmonary vein flow evaluation + based on proximal isovelocity surface area radius.

Additional file 2: Table 2. Adjusted hazard ratios for death or new heart failure of the final model for patients with myocardial infarction history (panel a), with definite diagnosis of coronary artery disease (panel b), and using MR derived left ventricle ejection fraction (panel c). $L G E=$ late gadolinium enhancement; $L V E F=$ left ventricle ejection fraction; $\mathrm{SPAP}=$ systolic pulmonary artery pressure.

\section{Competing interests}

The authors declare that they have no competing interests. 


\section{Acknowledgements}

We would like to thank Dr. Valeria Paliero, Ms Manuela Cattaneo, Mrs Cristina Spiaggi, and Mrs Miriam De Giorgi for their worthy collaboration on database maintenance.

\section{Author details}

'Divisione di Cardiologia, IRCCS Fondazione Salvatore Maugeri, via Maugeri 6, Pavia, Italy. ${ }^{2}$ Servizio di Diagnostica per Immagini, IRCCS Fondazione Salvatore Maugeri, via Maugeri 6, Pavia, Italy. ${ }^{3}$ Divisione di Cardiologia, Presidio Ospedaliero Sacro Cuore, Gallipoli, Italy. ${ }^{4}$ Direzione Scientifica Centrale, IRCCS Fondazione Salvatore Maugeri, via Maugeri 6, Pavia, Italy. ${ }^{5}$ Unità di Cardiologia Molecolare, IRCCS Fondazione Salvatore Maugeri, via Maugeri 6, Pavia, Italy. ${ }^{6}$ The Leon Charney Division of Cardiology, New York University School of Medicine, New York, USA. 'Dipartimento di Cardiologia, Università of Pavia, Pavia, Italy.

\section{Authors' contributions}

OC conceived and designed the study, evaluated CMR images, performed statistical analyses, interpreted results and wrote the manuscript. GM conceived and designed the study, evaluated CMR images. MP cooperated in the paper drafting. MF cooperated in the data acquisition. MC cooperated in the data acquisition. SA designed the study, cooperated in data analysed and interpreted results. PB critically cooperated in the results interpretation and revised statistics. CN critically revised the manuscript. MB gave the final approval. SGP gave the final approval. All authors read and approved the final manuscript.

Received: 18 October 2011 Accepted: 19 May 2012

Published: 19 May 2012

\section{References}

1. Kim RJ, Fieno DS, Parrish TB, Harris K, Chen EL, Simonetti O, Bundy J, Finn JP, Klocke FJ, Judd RM. Relationship of MRI delayed contrast enhancement to irreversible injury, infarct age, and contractile function. Circulation. 1999;100:1992-2002

2. Wu E, Judd RM, Vargas JD, Klocke FJ, Bonow RO, Kim RJ. Visualisation of presence, location, and transmural extent of healed Q-wave and non-Qwave myocardial infarction. Lancet. 2001;357:21-8.

3. Mahrholdt H, Wagner A, Holly TA, Elliott MD, Bonow RO, Kim RJ, Judd RM. Reproducibility of chronic infarct size measurement by contrastenhanced magnetic resonance imaging. Circulation. 2002:106:2322-7.

4. Kwong RY, Chan AK, Brown KA, Chan CW, Reynolds HG, Tsang S, Davis RB. Impact of unrecognized myocardial scar detected by cardiac magnetic resonance imaging on event-free survival in patients presenting with signs or symptoms of coronary artery disease. Circulation. 2006;113:2733-43.

5. Roes SD, Kelle S, Kaandorp TA, Kokocinski T, Poldermans D, Lamb HJ, Boersma E, van der Wall EE, Fleck E, de Roos A, Nagel E, Bax JJ. Comparison of myocardial infarct size assessed with contrast-enhanced magnetic resonance imaging and left ventricular function and volumes to predict mortality in patients with healed myocardial infarction. Am J Cardiol. 2007;100:930-6.

6. Kelle S, Roes SD, Klein C, Kokocinski T, de Roos A, Fleck E, Bax JJ, Nagel E. Prognostic value of myocardial infarct size and contractile reserve using magnetic resonance imaging. J Am Coll Cardiol. 2009;54:1770-7.

7. Kwon DH, Halley CM, Carrigan TP, Zysek V, Popovic ZB, Setser R, Schoenhagen P, Starling RC, Flamm SD, Desai MY. Extent of left ventricular scar predicts outcomes in ischemic cardiomyopathy patients with significantly reduced systolic function: a delayed hyperenhancement cardiac magnetic resonance study. JACC Cardiovasc Imaging. 2009;2:34-44.

8. Kim HW, Klem I, Shah DJ, Wu E, Meyers SN, Parker MA, Crowley AL, Bonow RO, Judd RM, Kim RJ. Unrecognized non-Q-wave myocardial infarction: prevalence and prognostic significance in patients with suspected coronary disease. PLoS Med. 2009;6:e1000057. Epub 2009 Apr 21.

9. Krittayaphong R, Boonyasirinant T, Chaithiraphan V, Maneesai A, Saiviroonporn P, Nakyen S, Thanapiboonpol P, Yindeengam A, Udompanturak S. Prognostic value of late gadolinium enhancement in hypertensive patients with known or suspected coronary artery disease. Int J Cardiovasc Imaging. 2010;26 Suppl 1:123-31.

10. Wu E, Ortiz JT, Tejedor P, Lee DC, Bucciarelli-Ducci C, Kansal P, Carr JC, Holly $T A$, Lloyd-Jones D, Klocke FJ, Bonow RO. Infarct size by contrast enhanced cardiac magnetic resonance is a stronger predictor of outcomes than left ventricular ejection fraction or end-systolic volume index: prospective cohort study. Heart. 2008:94:730-6.

11. van der Burg AE, Bax JJ, Boersma E, Pauwels EK, van der Wall EE, Schalij MJ. Impact of viability, ischemia, scar tissue, and revascularization on outcome after aborted sudden death. Circulation. 2003;108:1954-9.

12. Kim RJ, Wu E, Rafael A, Chen EL, Parker MA, Simonetti O, Klocke FJ, Bonow $\mathrm{RO}$, Judd RM. The use of contrast-enhanced magnetic resonance imaging to identify reversible myocardial dysfunction. $N$ Engl I Med. 2000:343:1445-53.

13. Rahimtoola SH. The hibernating myocardium. Am Heart J. 1989;117:211-21.

14. Pegg TJ, Selvanayagam JB, Jennifer J, Francis JM, Karamitsos TD, Dall'Armellina E, Smith KL, Taggart DP, Neubauer S. Prediction of global left ventricular functional recovery in patients with heart failure undergoing surgical revascularisation, based on late gadolinium enhancement cardiovascular magnetic resonance. J Cardiovasc Magn Reson. 2010;12:56.

doi:10.1186/1532-429X-14-29

Cite this article as: Catalano et al: Late gadolinium enhancement by cardiovascular magnetic resonance is complementary to left ventricle ejection fraction in predicting prognosis of patients with stable coronary artery disease. Journal of Cardiovascular Magnetic Resonance 2012 14:29.

\section{Submit your next manuscript to BioMed Central and take full advantage of:}

- Convenient online submission

- Thorough peer review

- No space constraints or color figure charges

- Immediate publication on acceptance

- Inclusion in PubMed, CAS, Scopus and Google Scholar

- Research which is freely available for redistribution 